\title{
Abstracts From the NCCN 2021 Virtual Annual Conference
}

\begin{abstract}
The following abstracts were accepted for presentation at the NCCN 2021 Virtual Annual Conference. Additional abstracts are available at JNCCN.org.
\end{abstract}

\section{YOUNG INVESTIGATOR AWARDS}

YIA21-001: A Modified Delphi Study to Refine Measures of End-of-Life Care Quality for Children With Cancer

Prasanna J. Ananth, MD, MPH ${ }^{1,2}$; Sophia Mun, $\mathrm{MPH}^{2}$;

Noora Reffat, $\mathrm{MPH}^{3}$; Soo Jung Kang, MD, $\mathrm{PhD}^{4}$; Sarah Pitafi ;

Xiaomei $\mathrm{Ma}, \mathrm{PhD}^{2,4}$; Cary P. Gross, $\mathrm{MD}^{2}$; and

Joanne Wolfe, MD, MPH $\mathrm{H}^{6,7,8}$

${ }^{1}$ Department of Pediatrics, Yale School of Medicine, New Haven, CT;

${ }^{2}$ Yale Cancer Outcomes, Public Policy and Effectiveness Research (COPPER) Center, New Haven, CT; ${ }^{3}$ University of Chicago Medicine,

Chicago, IL; ${ }^{4}$ Department of Chronic Disease Epidemiology, School of

Public Health, Yale University, New Haven, CT; ${ }^{5}$ Yale University, New

Haven, $C T$; ${ }^{6}$ Dana-Farber Cancer Institute, Boston, MA; ${ }^{7}$ Boston

Children's Hospital, Boston, MA; ${ }^{8}$ Harvard Medical School,

Boston, MA

This work was funded by the NCCN Foundation ${ }^{\circledR}$.

Background: There are no existing quality measures to define optimal end-of-life care (EOLC) for children with cancer, contributing to high healthcare utilization and variable palliative care provision. In a preceding study, we developed a set of 26 measures of EOLC quality. Our objective in the current study was to reach stakeholder consensus on which of these candidate quality measures to prioritize. Methods: We conducted an iterative crosssectional electronic survey, using a modified Delphi method. We convened a stakeholder panel, including interdisciplinary healthcare professionals, bereaved parents, and family advocates. In each of two rounds of surveys, participants were asked to rate quality measures on a 9-point Likert scale, based on perceived importance. Healthcare professionals were additionally asked to rate each measure on perceived feasibility. After each round, we computed median ratings on importance and feasibility of measurement. Open-ended responses following Round 1 were summarized to identify new quality measures to include in Round 2 . We retained quality measures that received a median score of $\geq 8$ in importance. Results: Twenty-five participants completed both rounds of the survey. In Round 1, participants were asked to rate 26 candidate EOLC quality measures. Following Round 1 , 9 quality measures were removed due to median importance scores $<8$. Six of the eliminated measures pertained to healthcare resource use. Two measures on advance care planning were then added in Round 2, based on participant comments. Following Round 2, 17 quality measures were ultimately retained (Table; next page). Quality measures related to symptom screening and receipt of palliative care consultation were rated highly, both in importance and feasibility. Quality measures related to communication were rated as highly important, yet less feasible. Measuring family members' receipt of psychosocial or bereavement services, and whether a patient's needs were heard by their healthcare team, were rated least feasible. Conclusions: We refined a candidate list of EOLC quality measures using the modified Delphi method. Childhood cancer stakeholders prioritized quality measures pertaining to patient-reported outcomes, deeming measures of healthcare resource use less important in this context. Future research should seek to implement these measures in real-world practice.

\section{BIOINFORMATICS/INFORMATION TECHNOLOGY SCIENCES}

BIO21-011: Oncology Provider Perspectives on Telemedicine for Patients With Cancer: A National Comprehensive Cancer Network (NCCN ${ }^{\circledR}$ ) Survey Amye Tevaarwerk, $\mathrm{MD}^{1}$; Travis Osterman, DO, $\mathrm{MS}^{2}$; Waddah Arafat, $\mathrm{MD}^{3}$; Jeffrey Smerage, $\mathrm{MD}, \mathrm{PhD}^{4}$; Fernanda C.G. Polubriaginof, $\mathrm{MD}, \mathrm{PhD}^{5}$; Tricia Heinrichs, $\mathrm{BS}^{6}$; Jessica Sugalski, MPPA ${ }^{6}$; and Daniel Martin, MD $^{7}$

${ }^{1}$ University of Wisconsin Carbone Cancer Center, Madison, Wl;

${ }^{2}$ Vanderbilt-Ingram Cancer Center, Nashville, TN; ${ }^{3}$ Harold C. Simmons Comprehensive Cancer Center, University of Texas Southwestern Medical Center, Dallas, TX; ${ }^{4}$ Rogel Comprehensive Cancer Center, University of Michigan, Ann Arbor, Ml; ${ }^{5}$ Memorial Sloan Kettering Cancer Center, New York, NY; ${ }^{6}$ National Comprehensive Cancer Network, Plymouth Meeting, PA; ${ }^{7}$ University of Washington Medical Center, Seattle, WA

Introduction: The use of telemedicine in the oncology (Onc) setting expanded after March 2020 due to the COVID-19 pandemic. Patients and providers report satisfaction with telemedicine within the context of acute pandemic needs (Darcourt et al, JOP, 2020). We assessed provider perspectives on the roles of phone- and video-based telemedicine in the Onc setting. Methods: The NCCN EHR Oncology Advisory Group formed a 


\section{YIA21-001 Table: Median Ratings of End-of-Life Care Quality Measure}

Quality Measure

-

Proportion of children with advanced cancer who are screened for physical symptoms (e.g., pain, nausea) during a healthcare visit in the last 30 days of life

Proportion of children with advanced cancer who have their symptoms satisfactorily managed during a healthcare visit in the last 30 days of life

Proportion of children with advanced cancer who receive psychosocial support

Proportion of parents of children with advanced cancer who receive bereavement services after their child's death

Proportion of parents of children with advanced cancer who receive direct communication about prognosis from a healthcare professional in the last 30 days of life

Proportion of parents of children with advanced cancer who communicate about their preferences for their child's care with a healthcare professional in the last 30 days of life

Proportion of adolescents with advanced cancer ( $>13$ years old) who communicate about their preferences for care with a healthcare professional in the last 30 days of life

Proportion of families of children with advanced cancer who receive anticipatory guidance about what to expect in the dying process

Proportion of children with advanced cancer who feel that their needs are heard by their care team

Proportion of children with advanced cancer whose location of death meets the child and/or family's preference

Proportion of children with advanced cancer whose end-of-life care is aligned with their documented goals

Proportion of children with advanced cancer who receive palliative care consultation prior to the last 30 days of life

Proportion of siblings of children with advanced cancer who receive psychosocial support

Proportion of parents of children with advanced cancer who receive psychosocial support

Proportion of children with advanced cancer who are enrolled in hospice services prior to death

Proportion of children with advanced cancer who receive home care to reduce travel time and time spent in the hospital

Proportion of children with advanced cancer who have a documented advance care plan in the last 30 days of life

\begin{tabular}{|c|c|c|}
\hline Domain & $\begin{array}{l}\text { Important } \\
\text { to Measure } \\
(n=25)\end{array}$ & $\begin{array}{l}\text { Feasible } \\
\text { to Measure } \\
(n=21)\end{array}$ \\
\hline Symptom Control & 9 [8-9] & 8 [5-9] \\
\hline Symptom Control & 9 [7-9] & $6[5-8]$ \\
\hline Interdisciplinary Care & 9 [7-9] & 7 [4-9] \\
\hline Interdisciplinary Care & 9 [7-9] & $5[2-9]$ \\
\hline Communication & 9 [7-9] & 7 [4-9] \\
\hline Communication & 9 [7-9] & 7 [3-9] \\
\hline Communication & 9 [7-9] & $6[2-9]$ \\
\hline Communication & 9 [7-9] & $6[2-8]$ \\
\hline Communication & 9 [6-9] & 5 [2-9] \\
\hline Meeting Patient Preferences & 9 [7-9] & 7 [2-9] \\
\hline Meeting Patient Preferences & 9 [7-9] & 6 [2-9] \\
\hline Interdisciplinary Care & 9 [3-9] & 8 [5-9] \\
\hline Interdisciplinary Care & 8 [6-9] & $5[2-7]$ \\
\hline Interdisciplinary Care & 8 [6-9] & 6 [2-9] \\
\hline Healthcare Resource Use & 8 [5-9] & 8 [5-9] \\
\hline Meeting Patient Preferences & 8 [5-9] & 6 [4-9] \\
\hline Meeting Patient Preferences & $8[5-9]$ & $8[5-9]$ \\
\hline
\end{tabular}

Workgroup to assess the state of Onc telemedicine, creating a 20-question survey distributed to all 30 NCCN Member Institutions. An NCCN member was responsible for emailing the survey to the Onc providers (surgery, hematology, gynecologic, medical and radiation oncology physicians \& APPs) at his/her institution. Results: $\mathrm{N}=1,038$ individuals from 26 institutions (institution response rate $87 \%$ ) responded 7-8/2020. Respondents were largely Onc physicians (58\%) with $>5$ years of practice experience $(72 \%)$. Few respondents had participated in telemedicine visits of any kind prior to the COVID-19 pandemic (19\%; $n=198 / 1038)$. At the time of the survey, most had participated in both telephone and videobased visits (84\%; $n=872 / 1038) ; 5 \%(n=52 / 1038)$ had participated in only phone-based visits and $9 \%(n=93 /$ 1038) had participated in only video-based visits. Only
$2 \%$ of respondents $(n=21 / 1038)$ reported not having conducted any telemedicine visits. The use of telemedicine was based on provider discretion $(88 \%$; $\mathrm{n}=727 / 826)$ and patient preference $(81 \% ; \mathrm{n}=669 / 826)$. Importantly, $94 \%(n=753 / 801)$ of respondents indicated rarely-to-never encountering adverse outcomes attributable to having had a telemedicine visit rather than an in-person visit. The Figure indicates how telemedicine visits compared to office visits for particular tasks commonly associated with Onc visits. Respondents $(n=796)$ estimated $33 \%$ of patients could safely be seen using video visits and $13 \%$ using phone visits post-pandemic. Challenges include lack of: 1) patient access to technology for video visits, 2) clinical workflows to support telemedicine, and 3) certainty about future insurance coverage. Conclusion: Based on Onc 

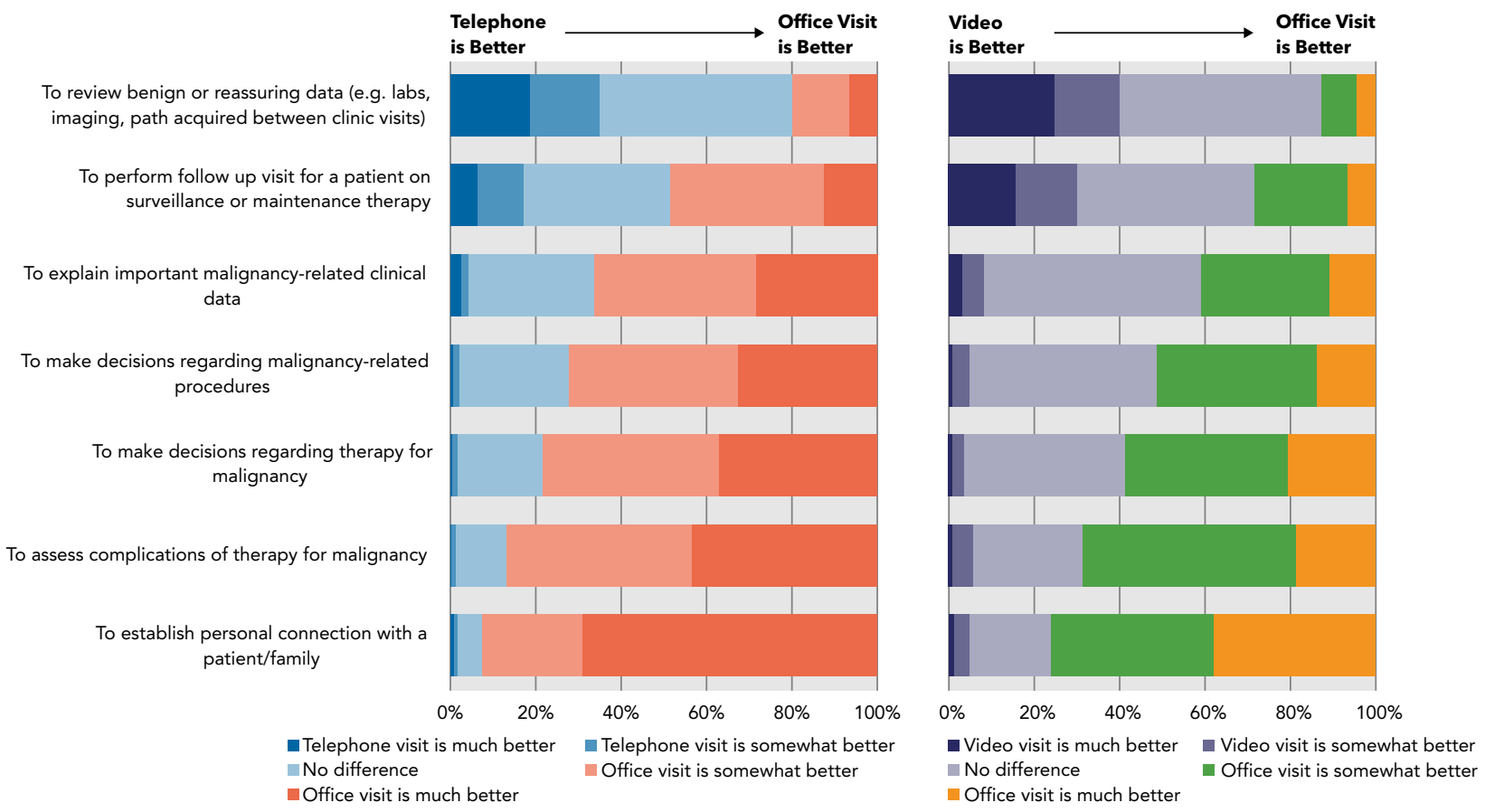

BIO21-011 Figure. Provider Perspective on How Telemedicine Compared to Office Visits for Particular Tasks Commonly Associated With Onc visits.

provider assessment, a substantial fraction of patient visits could be effectively, safely conducted using telemedicine post-pandemic. Overall, video visits were viewed more favorably than phone visits with greater utility over a wider range of clinical scenarios. Careful thought should be given to modifying regulations to maintain telemedicine for use post-pandemic.

\section{CLINICAL ONCOLOGY}

CLO21-016: Incidence, Risk Factors, and Prognostic Implications of Peri-Transplant Orthostatic Hypotension in Patients With Multiple Myeloma

Matthew Ho, MB BCh BAO, PhD ${ }^{1}$; Maria Moscvin, MD²;

Soon Khai Low, MD ${ }^{3}$; Sara Close, PA- $C^{4}$; Robert Schlossman, MD ${ }^{4}$; Jacob Laubach, $\mathrm{MD}^{4}$; Brett Glotzbecker, $\mathrm{MD}^{4}$;

Paul Richardson, MD; and Giada Bianchi, MD²

${ }^{1}$ Mayo Clinic, Rochester, MN; ${ }^{2}$ Brigham and Women's Hospital,

Boston, MA; ${ }^{3}$ Rochester General Hospital, Rochester, NY; ${ }^{4}$ Dana

Farber Cancer Institute, Boston, MA

Background: Orthostatic hypotension $(\mathrm{OH})$ is a wellrecognized phenomenon occurring in multiple myeloma (MM) patients undergoing autologous stem cell transplant (ASCT) that poses a morbidity and mortality threat due to increased risk of falls. Surprisingly, few studies have examined its incidence, risk and protective factors, and prognostic implications. Methods: This was a retrospective, single-center study of 226 consecutive newly diagnosed MM patients who were admitted for first ASCT between June 2012 to April 2014 at Dana Farber Cancer
Institute/Brigham and Women's Hospital, Boston, MA. Patients with AL amyloidosis were excluded. Orthostatic vital signs were checked on Monday, Wednesday and Friday. Median time to onset of $\mathrm{OH}$, progression free survival (PFS), overall survival (OS), and time to discharge were estimated using the Kaplan-Meier method. Univariable and multivariable logistic regression were used to investigate factors associated with the development of $\mathrm{OH}$ (Table). Results: Overall, 165/226 (73\%) patients were diagnosed with $\mathrm{OH}$ during the course of their hospital admission for ASCT. Fifty-one patients were found to have $\mathrm{OH}$ on the day of first orthostatic vitals check, making it impossible to distinguish whether $\mathrm{OH}$ was pre-existent or developed during the transplant admission. Excluding these 51 patients, 114/175 (65\%) patients developed $\mathrm{OH}$ during the peri-transplant period, at a median of 7 days post ASCT (range; 6-8). Of these patients, only eleven were found to have moderate to severe dehydration as defined by weight loss $\geq 5 \%$ body weight, suggesting $\mathrm{OH}$ could not be simply be explained by volume depletion. Multivariable analysis revealed three risk factors (white race, gabapentin, antihypertensives) and two protective factors (antihistamine, proton pump inhibitor) associated with the development of peri-transplant $\mathrm{OH}$ that were independent of significant fluid losses. Further, we found that $\mathrm{OH}$ did not significantly impact length of hospitalization, progression free and overall survival. Conclusions: New onset $\mathrm{OH}$ occurs frequently $(65 \%)$ during the peritransplant period in MM patients undergoing ASCT 


\begin{tabular}{|c|c|c|c|c|c|c|}
\hline \multicolumn{7}{|c|}{$\begin{array}{r}\text { CL021-016 Table: Univariable an } \\
\text { Hypotension }\end{array}$} \\
\hline \multirow[b]{3}{*}{ Variables } & \multicolumn{6}{|c|}{$\begin{array}{l}\text { (a) Post-admission (Days }-3 \text { to dismissal home) } \\
\text { (excluding the } 51 \text { patients who had OH on day of first orthostatic vitals check) }\end{array}$} \\
\hline & \multirow{2}{*}{$\begin{array}{l}\text { OH } \\
(n=113) \\
N(\%)\end{array}$} & \multirow{2}{*}{$\begin{array}{c}\text { No OH } \\
(n=61) \\
N(\%)\end{array}$} & \multicolumn{2}{|c|}{ Univariable analysis } & \multicolumn{2}{|c|}{ Multivariable analysis } \\
\hline & & & Risk Ratio $(95 \% \mathrm{Cl})$ & P-value & Risk Ratio $(95 \% \mathrm{Cl})$ & P-value \\
\hline White race & $106(93.8)$ & $50(83.3)$ & $3(1.1,8.4)$ & 0.034 & $5.3(1.3,21.4)$ & 0.019 \\
\hline$\geq \mathrm{CR}$ & $24(21.1)$ & $22(36.1)$ & $0.5(0.2,0.9)$ & 0.033 & $0.5(0.2,1.2)$ & 0.132 \\
\hline Antihistamine & $5(4.5)$ & $13(21.3)$ & $0.2(0.06,0.5)$ & 0.002 & $0.2(0.07,0.7)$ & 0.012 \\
\hline Gabapentin & $37(33)$ & $8(13.1)$ & $3.3(1.4,7.6)$ & 0.006 & $4.3(1.5,12)$ & 0.005 \\
\hline Proton pump inhibitor & $42(37.5)$ & $38(62.3)$ & $0.4(0.2,0.7)$ & 0.002 & $0.3(0.1,0.7)$ & 0.003 \\
\hline Antihypertensives & $59(52.7)$ & $22(36.1)$ & $2(1,3.7)$ & 0.038 & $2.7(1.2,6.1)$ & 0.021 \\
\hline \multirow[b]{3}{*}{ Variables } & \multicolumn{6}{|c|}{$\begin{array}{l}\text { (b) Post-admission (Days }-3 \text { to dismissal home) } \\
\text { (excluding the } 51 \text { patients who had OH on day of first orthostatic vitals check and } \\
11 \text { patients who had moderate to severe dehydration; i.e., body weight loss } \geq 5 \% \text { ) }\end{array}$} \\
\hline & \multirow{2}{*}{$\begin{array}{l}\mathrm{OH} \\
(n=103) \\
\mathrm{N}(\%)\end{array}$} & \multirow{2}{*}{$\begin{array}{l}\text { No OH } \\
(n=61) \\
N(\%)\end{array}$} & \multicolumn{2}{|c|}{ Univariable analysis } & \multicolumn{2}{|c|}{ Multivariable analysis } \\
\hline & & & Risk Ratio $(95 \% \mathrm{Cl})$ & P-value & Risk Ratio $(95 \% \mathrm{Cl})$ & P-value \\
\hline White race & $96(94.1)$ & $50(83.3)$ & $3.2(1.1,9.3)$ & 0.033 & $5.2(1.2,22.2)$ & 0.025 \\
\hline$\geq C R$ & $22(21.4)$ & $22(36.1)$ & $0.5(0.2,1)$ & 0.042 & $0.6(0.3,1.3)$ & 0.204 \\
\hline Antihistamine & $4(4)$ & $13(21.3)$ & $0.2(0.05,0.5)$ & 0.002 & $0.2(0.05,0.6)$ & 0.008 \\
\hline Gabapentin & $33(32.7)$ & $8(13.1)$ & $3.2(1.4,7.5)$ & 0.007 & $4.3(1.5,12.3)$ & 0.007 \\
\hline Proton pump inhibitor & $35(34.7)$ & $38(62.3)$ & $0.3(0.2,0.6)$ & 0.001 & $0.3(0.1,0.6)$ & 0.001 \\
\hline Antihypertensives & $54(53.5)$ & $22(36.1)$ & $2(1.1,3.9)$ & 0.033 & $2.8(1.2,6.7)$ & 0.021 \\
\hline
\end{tabular}

The table outlines variables associated with increased or decreased risk of developing $\mathrm{OH}$ in (a) patients who did not have $\mathrm{OH}$ on first check and (b) only patients who did not experience significant weight loss before $\mathrm{OH}$ and did not have $\mathrm{OH}$ on first check.

Bolded rows indicate variables significant in multivariable analysis.

(median time of onset of 7 days post ASCT). White race, use of gabapentin and antihypertensives were identified as risks factors, while use of antihistamines and proton pump inhibitor were identified as protective factors.

\section{CLINICAL ONCOLOGY}

CLO21-021: Impact on Survival of the Number of Lymph Nodes Resected in Patients With Esophageal Cancer Following Neo-Adjuvant Therapy

Jordan McDonald, BS ${ }^{1}$; Anupam Rishi, MD²; Sabrina Saeed, $\mathrm{MS}^{2}$; Rutika Mehta, MD, MPH ${ }^{2}$; David Pointer, MD²;

Jessica Frakes, $\mathrm{MD}^{2}$; Sarah Hoffe, $\mathrm{MD}^{2}$; Jacques Fontaine, $\mathrm{MD}^{2}$; and Jose Pimiento, $\mathrm{MD}^{2}$

${ }^{1}$ USF Health Morsani College of Medicine, Tampa, FL; ${ }^{2}$ Moffitt Cancer Center, Tampa, FL

Background: Esophagectomy with lymph node dissection following neoadjuvant therapy is the standard of care for resectable esophageal cancer. We explore whether a relationship exists between the examined lymph node (ELN) count and overall survival in those esophageal cancer patients receiving NAT in this study. Materials and Methods: In this IRB-approved retrospective study of 1200 patients with esophageal cancer treated between
1996-2019, we identified 786 patients consecutively treated with NAT followed by esophagectomy and had available ELN data. The data were analyzed to determine if there was a relationship between ELNs and survival. Overall survival (OS) was estimated using Kaplan-Meier method, and impact of ELN groups (by median and quartiles) were assessed using Cox proportional hazards regression and compared using log-rank test. Results: The cohort was predominantly male (83.6\%) and the median age at diagnosis was 64.7 years (range 28.2-86.3). Median ELN was 15 nodes (inter-quartile range 8-22). Median OS for the entire cohort was 42 months [95\% confidence interval (95\%CI) 35.8-48.3 months]. 5-year and 10 -year actuarial OS was 47.2 and $33.7 \%$, respectively. Patients with $\geq 16$ nodes had significantly improved OS compared with $\leq 15$ node group [median OS 49.8 (95\%CI 42.3-57.3) vs 33.4 months (95\% CI 25.4-41.3), respectively, $\mathrm{p}=0.03$ ]. 5 -year OS were $45.2 \%$ vs $49.2 \%$ for $\leq 15$ and $\geq 16$ LN groups, respectively. The upper quartile nodal group ( $\geq 22$ ELNs, median OS 47.9 months, CI 35.859.9) had longer median OS than the lower quartile LN group ( $\leq 8$ ELNs, median OS 36.1 months, 95\%CI 23.5-48.7). In multivariate analysis, nodal group remained significant $(\mathrm{p}=0.005)$ when compared with 
other established prognostic factors such as age at diagnosis, clinical and pathological stage and tumor regression grade. Conclusion: The number of lymph nodes removed in patients with esophageal cancer following neoadjuvant therapy is an independent prognostic factor for OS. Although there is no established minimum number of nodes removed after neoadjuvant chemoradiation, our results suggest the threshold should be set at 15 ELNs to most accurately stage EC post NAT and improve overall survival.

CLO21-025: Benefit of Deep Inspiratory Breath Hold for Right Breast Cancer When Regional Lymph Nodes Are Irradiated

Gabrielle W. Peters, MD; Sarah J. Gao, MD; Christin Knowlton, MD; Andrew Zhang, MD;

Suzanne B. Evans, MD, MPH; Susan Higgins, MD;

Lynn D. Wilson, MD, MPH; Nicholas Saltmarsh, CMD;

Martha Picone, CMD; and Meena S. Moran, MD

Yale School of Medicine, New Haven, CT

Background: While DIBH is routinely utilized for leftsided breast cancers, its benefits for $\mathrm{rBC}$ have yet to be established. We compared free-breathing (FB) and DIBH treatment plans for a cohort of $\mathrm{rBC}$ undergoing RNI to determine its potential benefits. Methods: rBC patients considered for RNI (IMNs, supraclavicular field, + / - axilla) from 10/2017-5/2020 were included in this analysis. For each patient, FB vs. DIBH plans were generated and dose volume histograms evaluated the following parameters: mean lung dose, ipsilateral lung V 20/V 5 (volumes of lung receiving $20 \mathrm{~Gy}$ and $5 \mathrm{~Gy}$, respectively); mean heart dose and heart V 5 (volumes of heart receiving 5 Gy); liver V 20 absolute/V 30 absolute (absolute volume of liver receiving $20 \mathrm{~Gy}$ and $30 \mathrm{~Gy}$, respectively), liver D max, and total liver volume irradiated (TVI liver). The dosimetric parameters were compared using Wilcoxon signed-rank testing. Results: 54 patients were eligible for analysis, comparing $108 \mathrm{FB}$ and DIBH plans. DIBH significantly decreased all lung and liver parameters: mean lung dose (19.7Gy to $16.2 \mathrm{~Gy}, \mathrm{p}<0.001)$, lung V $20(40.7 \%$ to $31.7 \%$, ( $<<0.001)$, lung V 5 ( $61.2 \%$ to $54.5 \%$, $\mathrm{p}<0.001)$, TV liver (1446cc vs 1264cc; $p=0.006$ ) liver D max (50.2 Gy vs $48.9 \mathrm{~Gy} ; \mathrm{p}=0.023$ ), liver $\mathrm{V} 20$ (78.8cc to $23.9 \mathrm{cc}$, $\mathrm{p}<0.001$ ) and liver V 30 (58.1cc to $14.6 \mathrm{cc}, \mathrm{p}<0.001$ ) when compared with FB. As expected for $\mathrm{rBC}$, DIBH use did not significantly improve heart parameters, although the $\mathrm{V} 5$ Heart trended on significance ( 1.25 to $0.6, \mathrm{p}=0.067$ ). Conclusion: This is the largest cohort to date analyzing DIBH for RNI-rBC. Our findings demonstrate significant improvement in all lung and liver parameters with DIBH, supporting its routine use for $\mathrm{rBC}$ pts being considered for comprehensive RNI. This study suggests that DIBH should be strongly considered in routine clinical practice for RNI-rBH to reduce lung and liver doses.
CLO21-027: Defining Optimal Lymph Node Dissection and Clinical Impact of Number of Harvested Lymph Nodes During Esophageal Resection for Early Stage Esophageal Cancer

Anupam Rishi, MD'; Jordan McDonald, MS²; Sabrina Saeed, BA ${ }^{1}$; Rutika Mehta, MD ${ }^{1}$; David Pointer, $\mathrm{MD}^{1}$; Jessica Frakes, MD ${ }^{1}$; Sarah Hoffe, MD ${ }^{1}$; Jacques Fontaine, $\mathrm{MD}^{1}$; and

Jose Pimiento, $\mathrm{MD}^{1}$

${ }^{1} \mathrm{H}$. Lee Moffitt Cancer Center \& Research Institute, Tampa, FL; ${ }^{2}$ Morsani College of Medicine, University of South Florida, Tampa, FL

Introduction: The optimal number of harvested lymph nodes (LNs) and its effect on clinical outcome remain controversial for patients with esophageal carcinoma. Objective of this study was to investigate the clinical impact of harvested LN with clinical outcome in patients with early esophageal cancer undergoing upfront esophagectomy. Materials and Methods: In this IRB-approved retrospective study of 1200 patients with esophageal cancer, we identified patients consecutively treated with upfront esophagectomy and had available lymphadenectomy data including LN count. The data were analyzed to determine if there was a relationship between LN count and outcome. Overall survival (OS) was estimated using Kaplan-Meier method, and impact of LN groups (dichotomized by median) were assessed using Cox proportional hazards regression and compared using log-rank test. Results: Between 1996-2019, 359 patients with esophageal cancer was treated with upfront esophagectomy. Median age was 65 years, and median follow-up duration was 63.7 months. Median number of harvested LN was 12 (inter-quartile range: 7-18). Median OS for the entire cohort was 99 months [95\% confidence interval (95\% CI) 73.9 - 124.1]. 5-year and 10 -year actuarial OS was 59.2 and $45.2 \%$, respectively. Patients dichotomized by median LN counts, patient with $>12$ nodes had significantly improved OS compared with $\leq 12$ node group [median OS 124.4 (95\%CI 86.1-162.2) vs 77.7 months (95\% CI 44.7-110.7), respectively, $\mathrm{p}=0.033$ ]. 10 -year OS were $40.7 \%$ vs $51.2 \%$ for $\leq 12$ and $>12$ LN groups, respectively. This statistical significance was lost when using $\leq 10$ harvested nodes as dichotomization cut-off $(p=0.2)$. Overall, 28/193 patients were upstaged from T0-T2/N0 to pathological stage IIb-IV after surgical resection. In multivariate analysis, nodal group remained significant $(\mathrm{p}=0.01)$ when compared with other established prognostic factors such as age at diagnosis $(p=0.2)$, clinical $(0.1)$, pathological stage $(p=0.000)$. Conclusion: The minimum number of harvested lymph nodes in patients with esophageal cancer following upfront esophagectomy affects stage migration and translates into overall survival benefit. Based on this study we recommend minimum number of 13 nodes harvested during lymphadenectomy. 


\section{CORRELATIVE/GENOMIC}

CGE21-031: Genomic Biomarker Testing, Treatments, and Survival Outcomes Among Patients With Advanced or Metastatic NSCLC in the US: A Retrospective Cohort Study

Yajun Zhu, MS, MBA; Yimei Han, MS; Naleen Raj Bhandari, PhD; and Lisa Hess, PhD

Eli Lilly and Company, Indianapolis, IN

Objectives: This study identified factors associated with genomic biomarker testing ("tested" vs "not tested") and examined the association of the receipt of treatment that is guided by genomic biomarker testing results with survival outcomes among patients with advanced/ metastatic NSCLC in the US. Methods: This retrospective observational study used Flatiron Health's electronic health records data (01JAN2015 to 31DEC2019) and included patients with non-squamous advanced/metastatic NSCLC. Eligible patients were categorized as "tested" if they received testing for genomic biomarkers including ALK, EGFR, ROS1, and/or BRAF prior to receiving first-line treatment and as "not tested" if otherwise. These patients were further categorized into four groups: (a) "tested-positive-concordant" (patients who were tested positive for a biomarker and received NCCNrecommended first-line treatment), (b) "tested-positivediscordant" (patients who were tested positive for a biomarker but did not receive NCCN-recommended first-line treatment), (c) "tested-but no positive results" (those who did not have a positive biomarker result), and (d) "not tested". Logistic regression and inverse weighting cox regression models were used to evaluate factors associated with testing and the association of testingguided treatment with survival outcomes, respectively. Results: 13,770 patients $(48.3 \%$ female; mean age (standard deviation) 68.0 (9.6) years) were included. Most patients $(82.6 \%)$ were tested for one of the four biomarkers before initiating first-line treatment. Factors associated with testing included smoking status, gender, body mass index, performance status, stage at initial diagnosis, year of advanced diagnosis, practice site and hospital's practice volume (all $\mathrm{p}<0.05$ ). After adjusting for all baseline covariates, the hazard of death was $17 \%$ lower in patients in the "tested-positiveconcordant" group compared those in the "testedpositive-discordant" group (adjusted hazard ratio $[\mathrm{aHR}]=0.83,95 \%$ confidence interval $[\mathrm{CI}]=0.80,0.86$ ). Likewise, the adjusted hazard of death was $50 \%$ higher in patients in the "not tested" group versus patients in the "tested-positive-discordant" group (aHR=1.50, $95 \% \mathrm{CI}=1.46,1.55)$. Conclusions: Real world data suggest that receipt of treatment that is guided by biomarker testing results is associated with improved survival outcomes in this patient population.

\section{EPIDEMIOLOGY/RISKS/PREVENTION}

EPR21-033: Testicular Cancer Trends and Disparities in the United States

Bohdan Baralo, MD; Mahati Paravathaneni, MD; Akhil Jain, MD; Eugene Choi, MD; and Rajesh Thirumaran, MD

Mercy Catholic Medical Center, Darby, Pennsylvania

Background: Healthcare disparities are well known for many types of the cancers. The last Surveillance, Epidemiology, and End Results Program (SEER) study of testicular cancer (TCa) used data collected up to 1999. These results were later confirmed in the separate studies for Asian and African Americans. The goal of our study is to evaluate the modern trends in incidence, stage on diagnosis and survival of patients of different races. Methods: SEER research data 18 registries, November 2019 Submission (2000-2017) was assessed for codes C60.1, C60.2, 60.3 to select patient with TCa. The analysis included the following groups defined by SEER: Hispanic and Non-Hispanic (Caucasians (White), African Americans (Black), Native Americans, Asians). Stages were defined as localized, regional and distant. Subjects with unknown survival or stage were removed. Linear regression, chi-square, log-rank test, Kaplan-Mayer method were used to estimate linear trends, odds (OR) and hazard ratios (HR). GraphPad Prism 8 (San Diego, CA) was used for analysis. Results: TCa is most frequently seen in patients 20-39 years. OR of having TCa were 0.2055 for African Americans, 0.8433 for Native Americans, 0.3357 for Asians, and 0.7932 for Hispanics when compared to Caucasians. Number of TCa cases in 2017 compared to 2000 increased by $7.24 \%$ for Caucasians males ( $\mathrm{p}=0.0008), 13.91 \%$ for Asians $(\mathrm{p}=0.0002), 33.87 \%$ for African Americans $(p=0.0238), 46.67 \%$ for Hispanic $(p<0.0001)$ and 87.97 Native Americans $(p=0.0145)$. African Americans $(p=0.0064)$ and Asian $(p=0.0006)$ groups showed increased incidence of localized TCa. Native Americans $(p=0.0013)$ and Caucasians $(p=0.0079)$ group showed an increased incidence of regional TCa. Hispanic group showed growth of TCa among all stages$\mathrm{p}<0.0001$ for local; $<0.0001$ for regional; 0.0002 for distant stage (Figure). HR for overall and cancer-specific survival (CSS) showed that African American, Asian and Hispanic population had worse outcomes compared to Caucasians (CSS for African American 2.25, Asian 1.302, Hispanic 1.122). Conclusions: The TCa cases are growing among all races. Caucasians remains the group with the highest likelihood of developing TCa. Growth is observed not only among localized stage at the time of presentation, but also regional and distant. The growth of TCa cases group was most significant in Hispanic group among all stages. Caucasians males had the best survival compared to other races, except Native American. 

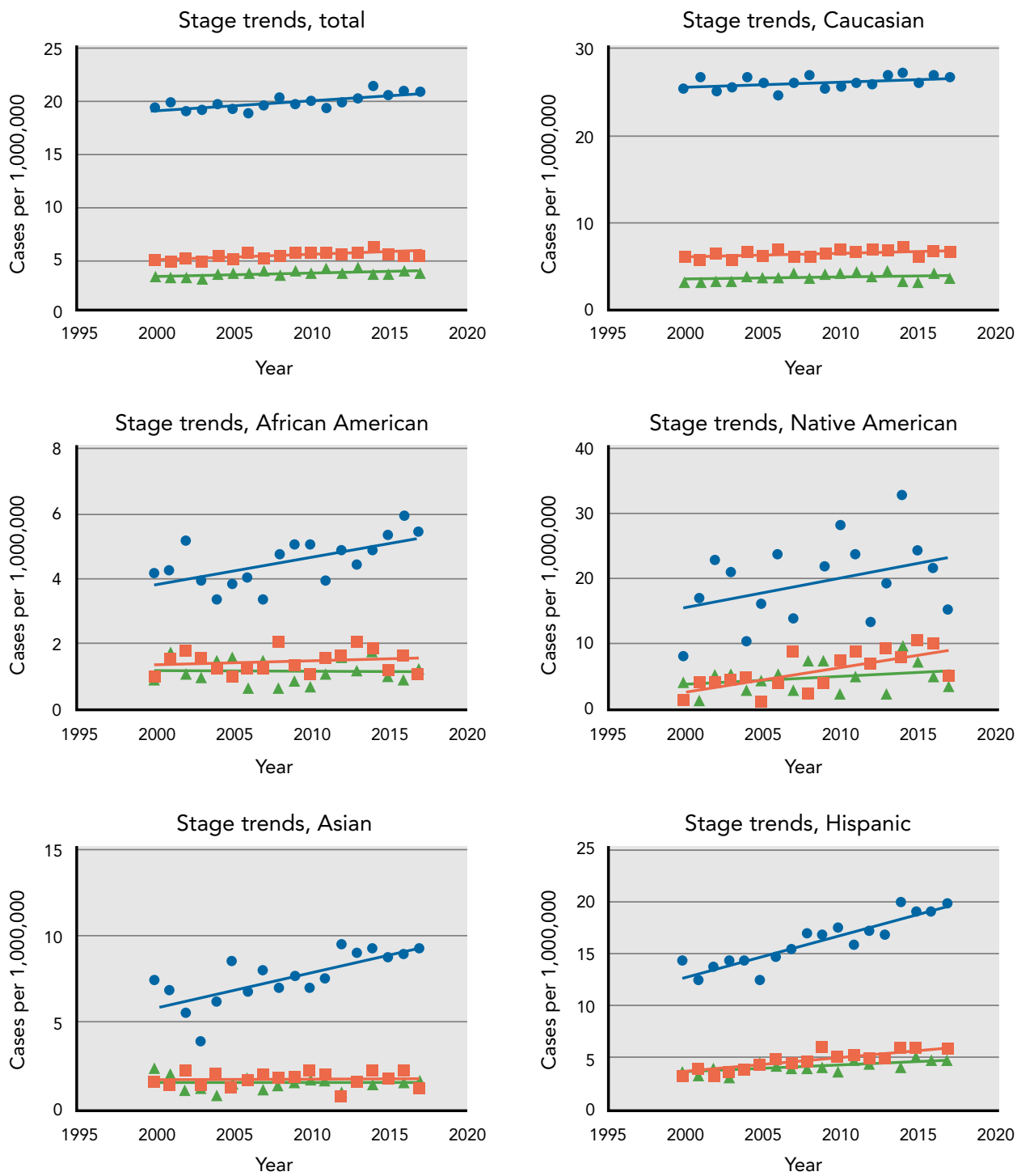

EPR21-033 Figure.

\section{EPR21-040: Prescribing Patterns at the End of Life in Lung Cancer \\ Catherine Yip, $\mathrm{BS}^{1}$; Evangelia Valilis, $\mathrm{MD}, \mathrm{MPH}^{1}$; \\ Laura Prichett, $\mathrm{PhD}, \mathrm{MHS}^{1}$; Catherine Burdalski, PharmD, BCOP${ }^{1}$; and Josephine Feliciano, MD ${ }^{2}$ \\ ${ }^{1}$ Johns Hopkins University School of Medicine, Baltimore, MD; ${ }^{2}$ The Sidney Kimmel Comprehensive Cancer Center at Johns Hopkins, Baltimore, MD}

Background: Studies have found that monthly cancer treatment costs are often stable until the final month before death when costs increase regardless of survivorship status. Hospitalization is a major factor, but this also begs the question of whether hail-Mary approaches are being sought at the end of life. A short interval between the last chemotherapy and death is often viewed as an indicator of low-quality care. As such, we aim to evaluate prescribing patterns at the end of life in patients with lung cancer at Johns Hopkins. Methods: Patients with histologically confirmed small cell and NSCLC who received treatment at Johns Hopkins and died of lung cancer from 2013 to 2018 were identified from the Maryland State Health Department Database. Data including gender, race, marital, insurance, smoking statuses, zip code as an income proxy, ECOG performance status, and lines and types of treatment were collected through EMR chart review. Multivariate logistic regression was used to evaluate for association between these variables and outcome of receiving any treatment, focusing on within 30 days of death. Results: 1146 patients were identified, 613 (53\%) of whom were male and $533(47 \%)$ were female. 764 (67\%) were Caucasian, 298 (26\%) were Black, and 81 (7\%) were Asian/Pacific Islander. 641 patients (56\%) 
were from zip codes with median income $\$ 50,000$ to $\$ 100,000$ and 353 (31\%) less than \$50,000. 460 (40\%) patients had private insurance, 422 (37\%) Medicare with secondary insurance, 145 (13\%) Medicare only, 68 (6\%) Medicare with Medicaid, and 27 (2\%) Medicaid only. $582(51 \%)$ patients received treatment within 30 days of death. Of 209 SCLC patients, 39 (19\%) were treated within 30 days of death. 2 received oral targeted therapy, 9 oral chemotherapy, 25 IV chemotherapy, and 3 IV immunotherapy. Of 937 patients diagnosed with metastatic NSCLC, 135 (14\%) were treated within 30 days of death. 48 received oral targeted therapy, 4 oral chemotherapy, 61 IV chemotherapy, and $21 \mathrm{IV}$ immunotherapy. Income $>\$ 100,000$ was significantly associated with receiving therapy for NSCLC within 30 days of death $(p=.037)$. Conclusions: About half of patients in this study received some treatment in the last 30 days of life. This occurred independent of insurance status but was significantly associated with income over $\$ 100,000$ for NSCLC. This suggests that a substantial number of patients are still being treated shortly before their death, a practice that warrants further exploration.

\section{OUTCOMES AND HEALTH SERVICES RESEARCH}

HSR21-059: Telehealth is Here-Are Patients Ready for It? An Analysis of Technological Knowledge in

\section{Cancer Patients}

Jeremie M.P. Lever, PhD; Ahmer Irfan, MD; Mona Fouad, MD;

Barry Sleckman, MD, PhD; J. Bart Rose, MD;

Thomas N. Wang, MD, PhD; Daniel I. Chu, MD; and

Sushanth Reddy, MD

O'Neal Comprehensive Cancer Center, University of Alabama at Birmingham School of Medicine, Birmingham, AL

Background: The COVID-19 pandemic has led to the rapid expansion of telehealth use. Telehealth has the potential to improve access for underserved populations who live far distances from well-equipped medical centers. This healthcare modality will likely be particularly important for patients with cancer. We hypothesized that there are disparities in the resources available to utilize telehealth and sought to study patient access and knowledge of associated technologies. Methods: We conducted a single-center cross-sectional survey study of patients at an NCCN-designated comprehensive cancer center over a two-month period. Demographics, education, internet, and cell phone access were assessed. Participant technological knowledge was determined with a validated 10-question quiz of terms regarding computers and the internet. Income was extrapolated using IRS data for individual zip codes. Analysis was performed using either an ordered logistic regression or mixed model ordered logistic regression (Stata SE
16.1). Results: There were 344 survey responses (rate $64.3 \%$ ). Mean age of the respondents was 57.5 years, $70.0 \%$ were women, $67.3 \%$ were Caucasian, and $25.4 \%$ were Black. Many patients (35.0\%) did not attend college, and $5.0 \%$ had not finished high school. The median estimated income was $\$ 45,820$. Ninety-six patients $(30.0 \%)$ did not have adequate internet access for telehealth use with $6.5 \%$ not having any internet access at all. Participants scored an average of $57.4 \%$ correct on the technology knowledge quiz. In unadjusted analysis, technological knowledge was predicted by age (Fig A), race (Fig B), income (Fig C), education level (Fig D), and cell phone type (Fig E). The type of home internet access and was not predictive of quiz score. Age, race, education level, and cell phone type remained significantly predictive of technological knowledge in multivariable analysis (Fig F). Home internet access ( $p$ $=0.416)$ and median income $(p=0.109)$ were not significantly associated with quiz score. Conclusions: In our sample, nearly one-third of cancer patients did not have adequate internet access for telehealth. There were significant disparities in technological knowledge among cancer patients which were associated with demographic characteristics. This study provides information for identifying patients that may have barriers to successful utilization of telehealth.

\section{HSR21-071: Healthcare Utilization Between Blood and Solid Cancer Survivors and Implications for Financial Toxicity: Results From the National Health Interview Survey, 2009-2018 \\ Christopher T. Su, MD, MPH, and Minal R. Patel, PhD, MPH University of Michigan, Ann Arbor, MI}

Background: Frequent care utilization leads to escalating medical costs, which can result in financial toxicity in cancer survivors. Although these differences are striking when comparing individuals with and without cancer, similar differences have not been studied across cancer types. We aim to examine differences in healthcare utilization and financial hardship between blood and solid cancer survivors. Methods: Blood and solid cancer survivors diagnosed within the last 10 years were identified from 2009-2018 National Health Interview Survey data. Survivor-reported responses assessing care utilization and financial hardship were analyzed with univariate tests, followed by multivariable regressions. All analyses were weighted and stratified to create nationallyrepresentative estimates. Results: 724 and 11,553 respondents were identified, representing 0.25 and 3.9 million blood and solid cancer survivors nationally. Mean time since diagnosis were 3.3 and 3.7 years for blood and solid cancer survivors, respectively $(p=0.13)$. Blood cancer survivors had worse general health status (odds ratio [95\% confidence interval]: 
A

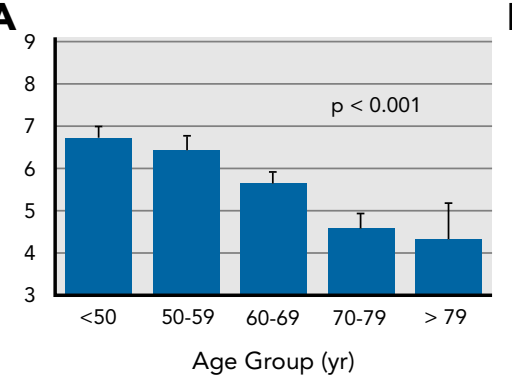

D

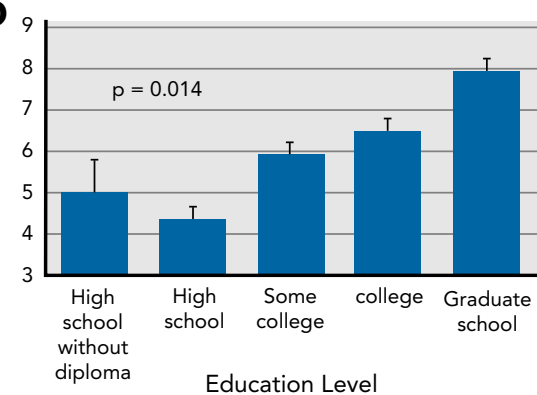

$\mathbf{F}$

\begin{tabular}{lll}
\hline Variable & Z score & P value \\
\hline Age & -2.2 & $\mathbf{0 . 0 2 8}$ \\
Race & $\mathbf{2 . 8 2}$ & $\mathbf{0 . 0 0 5}$ \\
\hline Income & 1.60 & 0.109 \\
\hline Highest level of education & $\mathbf{3 . 9 2}$ & $<\mathbf{0 . 0 0 1}$ \\
\hline Cell phone type & $\mathbf{- 2 . 3 4}$ & $\mathbf{0 . 0 1 9}$ \\
\hline Home internet access & -0.81 & 0.416 \\
\hline
\end{tabular}

B

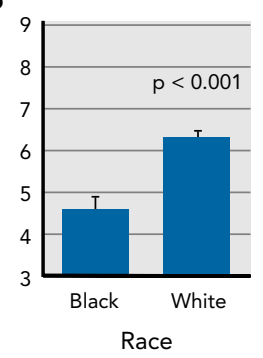

C

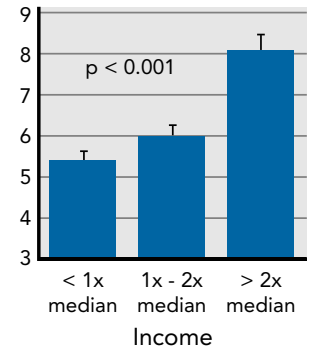

E

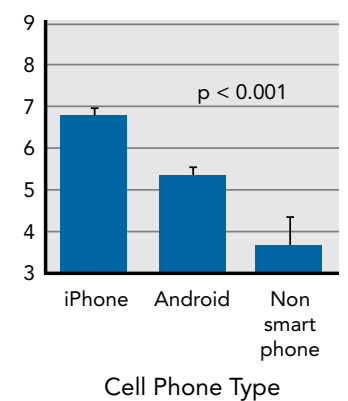

HSR21-059 Figure. Technology knowledge quiz score.

Bold indicates statistically significant $P$ value.

0.63 [0.51-0.77], $\mathrm{p}<0.0001$ ), received more healthcare (1.34 [1.10-1.63], $\mathrm{p}<0.01$ ), had increased office visits to medical providers (1.31 [1.10-1.56], $\mathrm{p}<0.01)$ and medical specialists (1.61 [1.27-2.04], $\mathrm{p}<0.0001)$, compared to solid cancer survivors. Additionally, blood cancer survivors were more likely to go to the emergency department because they were sent by a physician (1.77 [1.18-2.66], $\mathrm{p}<0.01$ ), the hospital is the only place that could help (1.93 [1.12-3.32], $\mathrm{p}=0.02)$, and their symptoms were too serious for outpatient care (1.60 [1.02-2.52], $\mathrm{p}=0.04)$. Furthermore, blood cancer survivors were more likely to be admitted overnight and spend more nights in the hospital (both $\mathrm{p}<0.0001$ ). Increased care utilization was associated with increased inability to afford necessary medical care, medications, specialist care, and pay medical bills (all $\mathrm{p}<0.0001$ ). However, these relationships were not significant based on cancer type. Discussion: Blood cancer survivors utilize more healthcare compared to solid cancer survivors. More care utilization is significantly associated with increased financial hardship. Although no differences in financial hardship by cancer type were observed from our data, more work needs to be done to understand financial consequences on blood cancer patient/families that result from higher care utilization.

\section{HSR21-075: CancerSupportSource ${ }^{\circledR}$-Caregiver:}

Psychometric Properties of 2-Item Depression and Anxiety Risk Screening Measures for Informal Cancer Caregivers

Alexandra K. Zaleta, PhD; Melissa F. Miller, PhD, MPH;

Erica E. Fortune, PhD; Kelly A. Clark, MA; and

Elissa C. Kranzler, PhD

Cancer Support Community, Research and Training Institute, Philadelphia, PA

Background: Caring for loved ones with cancer can be burdensome and emotionally demanding; screening for caregiver depression and anxiety can mitigate the risks of under-identifying and undertreating these conditions. CancerSupportSource ${ }^{\circledR}$-Caregiver (CSSCG) is a 33-item multidimensional distress screening and referral program with items related to depression (feeling sad or depressed; feeling lonely or isolated) and anxiety (feeling nervous or afraid; worry about the future and what lies ahead). We evaluated the 
psychometric performance of CSS-CG depression (CSSD2) and anxiety (CSS-A2) risk screening subscales among community-based family and informal caregivers. Methods: 328 cancer caregivers enrolled in Cancer Support Community's Cancer Experience Registry and rated CSSCG items ( $0=$ Not at all; $4=$ Very seriously), PROMIS-29 v2.0 Depression \& Anxiety Short Forms 4a (PROMIS-D; PROMISA), and PHQ-2 Depression \& GAD-2 Anxiety. We calculated Pearson correlations; area under the curve (AUC) with PROMIS-D T-score $\geq 60$ and PHQ-2 $\geq 3$ to flag depression risk, and PROMIS-A T $\geq 62$ and GAD- $2 \geq 3$ for anxiety risk; and calculated sensitivity/specificity and positive/negative predictive values (PPV/NPV) with cut-scores of $\geq 3$ for each CSS-CG risk scale. Results: $55 \%$ of caregivers were identified as at risk for clinically significant depression, $68 \%$ for anxiety. Regarding comorbid risk: $50 \%$ both, $4 \%$ depression only, $18 \%$ anxiety only. CSS-D2 was correlated with PROMIS-D ( $\mathrm{r}=.76$; $\mathrm{p}<.001$ ), $\mathrm{AUC}=.879$, sensitivity $=.95 /$ specificity $=.67, \mathrm{NPV}=.96$ / $\mathrm{PPV}=.60$; and also with PHQ-2 $(\mathrm{r}=.61 ; \mathrm{p}<.001), \mathrm{AUC}=.868$, sensitivity $=.93 /$ specificity $=.63, \mathrm{NPV}=.95 / \mathrm{PPV}=.54$. CSS-A2 was correlated with PROMIS-A ( $\mathrm{r}=.79 ; \mathrm{p}<.001), \mathrm{AUC}=.879$, sensitivity $=.95 /$ specificity $=.54, \mathrm{NPV}=.93 / \mathrm{PPV}=.63$; and also with GAD-2 $(\mathrm{r}=.62 ; \mathrm{p}<.001), \quad \mathrm{AUC}=.867$, sensitivity $=$ $.98 /$ specificity $=.51, \quad \mathrm{NPV}=.97 / \mathrm{PPV}=.43$. Conclusions: CSS-CG depression and anxiety risk subscales demonstrated good concurrent validity and sensitivity among cancer caregivers, with lower specificity. Embedding clinical risk screening within the broader CSS-CG screening and referral program provides flexibility in assessing unmet caregiver needs and clinical distress while minimizing burden. In implementation of CSS, follow-up procedures should be well-defined for individuals who screen positive. Future work will evaluate psychometric support for a shortened CSS-CG tool and should examine CSS-CG risk scales against structured diagnostic interviews.

\section{QUALITY IMPROVEMENT}

QIM21-091: Patterns of Health Portal Usage Among Patients With Cancer During the Year of Diagnosis: Results From the UWCCC Survivorship Program

Rebecca P. Luoh, BS ${ }^{1}$; Amye J. Tevaarwerk, MD ${ }^{1,2}$;

Thevaa Chandereng, $\mathrm{PhD}^{3}$; Elena M. Smith, $\mathrm{MS}^{4}$;

Cibele Carroll, MD, MPH ${ }^{2}$; Hamid Emamekhoo, $\mathrm{MD}^{1,2}$; and

Mary E. Sesto, PT, PhD ${ }^{1,2}$

${ }^{1}$ University of Wisconsin School of Medicine and Public Health, Madison,

WI; ${ }^{2}$ University of Wisconsin Carbone Cancer Center, Madison, WI;

${ }^{3}$ Columbia University, New York, NY; ${ }^{4}$ UWHealth, Madison, WI
Background: Online patient portals are important tools to help patients with cancer participate in their health care processes. Portals provide patients with real-time access to their data, and actions such as communicating with providers, assessing medical exam results, and reviewing the medical plan can improve self-management of care. All of this may assist in managing health. It may be especially pertinent for patients with cancer considering the complexity of information and tasks to cope with, especially during diagnosis and active treatment. Thus, understanding how patients with cancer use portals is crucial to support care engagement. Our objective was to evaluate portal usage comparing cancer vs noncancer purposes. Methods: We evaluated portal usage, demographic, and cancer-related data from patients with solid tumors, seen between 2015 and 2019, at the Medical Oncology Departments of a comprehensive cancer center. Statistical analysis was performed with paired Wilcoxon rank-sum test. A p-value of 0.05 was considered statistically significant. The study was exempted from the Institutional Review Board review. Results: 5950 patients meet the inclusion criteria. Mean age was 64 (SD \pm 13 ), $54 \%(n=3185)$ were female, $94 \%(n=5587)$ white, $72 \%$ $(\mathrm{n}=4271)$ resided in urban area (RUCC $1-3)$, and $44 \%$ $(n=2595)$ were Medicaid/Medicare beneficiaries. Twenty seven percent $(n=1620)$ had localized disease, $21 \%$ $(n=1256)$ were metastatic. Nearly $34 \%(n=2052)$ of the population did not have an active account. The most common functionalities used by patients with an account, during the year following the diagnosis were "View past and upcoming visits", "View results", and "View and act on messages", in descending order. Patients were less likely to send cancer messages compared to noncancer messages $(\mathrm{P}<0.0001)$. Patients were also less likely to view results for cancer care purposes versus noncancer $(\mathrm{P}<0.0001)$. Discussion: The volume of portal messages sent was low, as well as the percentage of tests viewed; even so, patients were less likely use portals to send cancer-related messages or view cancer-related tests. Moreover, over one-third of patients did not have an active account. Thus, designing portal features and functionalities to support cancer patient engagement and facilitate self-management of care is needed. Conclusions: Patient portals have a promising, but not wellexplored potential to improve cancer patients' selfmanagement of care. 